\title{
Avaliando o Impacto da Estratégia de Segurança do Paciente Implantada em uma Unidade de Clínica Médica de um Hospital Universitário sob a Perspectiva da Dimensão da Atenção à Saúde
}

\author{
Assessing the impact of Patient Safety strategy implemented in a Clinical Unit of a University \\ Hospital under the Perspective of Health Care Dimension
}

\section{Joseilda Alves da Silva', Flávia Cristina Morone Pinto²}

1. Graduanda do Curso de Saúde Coletiva, do Centro Acadêmico de Vitória (CAV), da Universidade Federal de Pernambuco (UFPE), Vitória de Santo Antão. Pernambuco. Brasil.

2. Enfermeira. Doutora em Ciências (UERJ). Professora Adjunta do Curso de Saúde Coletiva, do Centro Acadêmico de Vitória (CAV), da Universidade Federal de Pernambuco (UFPE), Vitória de Santo Antão. Pernambuco. Brasil.

\section{RESUMO}

Considerando a magnitude do tema Segurança do Paciente (SP) e sua relevância na dimensão do cuidado em saúde e na qualidade da assistência. Este estudo tem por objetivo analisar as estratégias de segurança do paciente implantadas na unidade de clínica médica (UCM) de um hospital de ensino em Pernambuco. Foi utilizada a metodologia quanti-qualitativa de natureza descritiva, por meio da análise dos indicadores do Plano de Segurança do Paciente (PSP) da Instituição. Para coleta dos dados utilizou-se um formulário adaptado da EBSERH (Empresa Brasileira de Serviços Hospitalares). Foi estabelecido o Índice de Conformidade (IC) ideal $\geq 80 \%$ para todos os indicadores descritos, ou seja, o percentual de adequação que reflete a segurança em relação à assistência prestada. Quanto a Cultura Organizacional observou-se um IC de $60.97 \%$. Quanto aos Processos de Gestão de Risco teve um IC de 60,0\%; e, quanto ao Núcleo de Segurança do Paciente na UCM, um IC de 56,25\%. Conclui-se que há a necessidade da estruturação de medidas que assegurem a implantação de uma cultura voltada para a segurança do paciente, identificando erros, adequando processos e promovendo melhorias contínuas, na busca pela qualidade da assistência à saúde.

Palavras-chave: Gestão da Qualidade. Segurança do Paciente. Gestão de Riscos. Cultura Organizacional. 
The magnitude of the patient safety (SP) issue and its relevance in the dimension of health and quality of care it is essential to the development of studies to assess the relationship between health care processes and outcomes from it, securing to improve quality assistance in the hospital network. This study aimed to analyze the patient safety strategies implanted in the medical clinic unit of a teaching hospital in Pernambuco. Was used a quantitative methodology of a descriptive nature, through the analysis of Patient Safety Plan indicators (PSP). For data collection was used an adapted form of EBSERH (Brazilian's Company of the Hospital Services). It was established the ideal Compliance Index $(\mathrm{Cl}) \geq 80 \%$ for all indicators described, i.e. the adequacy percentage that reflects the safety for the assistance provided. The Organizational Culture there was an IC of $60.97 \%$. As for the risk management processes had a Cl 60.0\%; and as the Patient Safety Center at UCM, an IC of $56.25 \%$. In conclusion, there is a need of structuring measures to ensure the implementation of a culture focused on patient safety, identifying errors, adapting processes and promoting continuous improvement of the health care quality and patient safety.

Keywords: Quality Management. Patient Safety. Risk Management. Organizational Culture.

\section{INTRODUÇÃO}

Nos últimos anos o tema Segurança do Paciente (SP) vem sendo amplamente discutido na busca da melhoria da qualidade da assistência a saúde, pois eventos adversos relacionados à assistência são frequentes, tornando a SP como um componente crítico de melhoria da qualidade do cuidado de saúde em todo o mundo, visto que constitui globalmente um grave problema de saúde pública ${ }^{1}$. Segundo a Organização Mundial de Saúde (OMS) a segurança do paciente é definida como a redução, a um mínimo aceitável, do risco de dano desnecessário associado ao cuidado de saúde².

O relatório sobre erros relacionados com a assistência à saúde "Errar é humano: construindo um sistema de saúde mais seguro"3, apontando a elevada ocorrência de eventos adversos nos hospitais, sendo 44.000 a 98.000 óbitos evitáveis por ano nos EUA (Estados Unidos da América) serviu como um marco para mobilização do movimento mundial sobre a SP, tendo como seu primeiro desafio global infecções relacionadas à assistência.

A partir da década de 2000, a segurança do paciente entra para a agenda de pesquisadores de todo o mundo e passa a ser internacionalmente reconhecida como uma dimensão fundamental da qualidade em saúde. Reconhecendo a magnitude do problema da segurança do paciente a nível global, a OMS estabeleceu em 2004 a Aliança Mundial para a Segurança do Paciente (World Alliance for Patient Safety). O propósito dessa iniciativa foi definir e identificar prioridades na área da segurança do paciente em diversas partes do mundo e contribuir para uma agenda mundial para a pesquisa no campo'1.

De acordo com Aline, Bueno \& Fassarella (2011), no Brasil, pesquisas nesta área surgiram no início da década de 2000, influenciados pelo panorama global ${ }^{4}$. Quase duas décadas já se passaram e ainda continua incipiente o processo de SP e uma assistência de qualidade nas unidades de saúde da rede publica.

Como resposta aos fatos e consequências decorrente de erros humanos a Joint Commission International ( $\mathrm{JCl}$ lançou em 2011 as seis Metas Internacionais para a Segurança do Paciente 
(International Patient Safety Goals - IPSG) ${ }^{5}$, onde considera-se que avaliar a SP no hospital permite identificar e gerir antecipadamente questões relevantes de segurança nas rotinas de trabalho.

Este estudo teve como objetivo analisar as estratégias de segurança do paciente implantadas na unidade de clínica médica de um hospital de ensino em Pernambuco, quanto à eficiência na detecção, prevenção e atenuação de erros e eventos adversos no setor, sob a perspectiva da dimensão do cuidado de saúde.

\section{METODOLOGIA}

\section{Desenho do estudo}

Estudo descritivo e abordagem quanti-qualitativa, por meio da análise dos indicadores do Plano de Segurança do Paciente (PSP). Adicionalmente foi realizado um levantamento bibliográfico em base de dados de abrangência nacional e internacional, LILACS (Literatura Latino Americana de Ciências de Saúde), Scielo (Scientific Eletronic Library Online), CQH (Compromisso com a Qualidade Hospitalar) PROQUALIS e MedLine (Medical Literature Analysis and Retrieval System Online), no período entre março a outubro de 2016 com os seguintes descritores: Segurança do paciente; dimensão do cuidado; Gerenciamento de Risco; Ferramentas para a Gestão da Qualidade. O objetivo deste levantamento foi fundamentar o assunto sem a intenção de esgotá-lo.

\section{Local do Estudo}

O cenário do estudo foi uma Unidade de Clínica Médica (UCM) de um Hospital Universitário de Ensino Federal, um órgão suplementar à Universidade, e gerenciado desde 2013, pela Empresa Brasileira de Serviços Hospitalares (EBSERH) e que atua como hospital-escola.

\section{Instrumento de Pesquisa e coleta de dados}

O plano de segurança do paciente instituído neste Hospital, para a UCM foi analisado. A partir desta análise foram definidos os indicadores prioritários a serem avaliados, quanto aos processos de segurança do paciente.

Foi utilizado um formulário previamente adaptado do Roteiro para diagnósticos das filiais EBSERH.

De acordo com este roteiro, os parâmetros usados para auxiliar esta análise foram abordados em três grandes áreas: Cultura Organizacional; Funcionamento do Núcleo de Segurança do Paciente e Gestão de Riscos.

A seguir, na Tabela 1, estão descritas as dimensões mensuráveis pelo instrumento com o objetivo de auxiliar o entendimento no diagnostico situacional, levando-o a construção do PI com foco na Segurança do Paciente da Unidade de Clínica Médica. 


\begin{tabular}{|c|c|c|}
\hline & Mensuráveis & \\
\hline \multirow[t]{2}{*}{$\begin{array}{l}\text { Cultura } \\
\text { organizacional }\end{array}$} & Protocolos & $\begin{array}{l}\text { - Identificação correta do paciente } \\
\text { - Guarda e administração de medicamentos } \\
\text { - Prevenção de quedas } \\
\text { - Prontuários: checagem e registros } \\
\text { - Prevenção de úlceras de pressão } \\
\text { - Prevenção de quedas } \\
\text { - Prevenção de infecções } \\
\text { - Administração de sangue e hemocomponentes } \\
\text { - Transferência de pacientes } \\
\text { - Higienização das mãos } \\
\text { - Gerenciamento de resíduos do serviço de saúde } \\
\text { - Uso seguro de equipamentos }\end{array}$ \\
\hline & Segurança & $\begin{array}{l}\text { - Riscos inerentes ao processo de trabalho } \\
\text { - Gerenciamento de risco }\end{array}$ \\
\hline \multirow[t]{4}{*}{ Gestão de riscos } & Tecnovigilância & - Realiza ações de tecnovigilância \\
\hline & Farmacovigilância & $\begin{array}{l}\text { - Erro de medicação } \\
\text { - Reações adversas } \\
\text { - Efetividade terapêutica } \\
\text { - Queixas técnicas } \\
\text { - Uso off label }\end{array}$ \\
\hline & Hemovigilância & $\begin{array}{l}\text { - Reações transfusionais } \\
\text { - Demais incidentes }\end{array}$ \\
\hline & Monitoramento & $\begin{array}{l}\text { - Indicadores de acompanhamento } \\
\text { - Média mensal de notificação }\end{array}$ \\
\hline \multirow[t]{2}{*}{$\begin{array}{l}\text { Funcionamento } \\
\text { dos núcleos de } \\
\text { segurança do } \\
\text { paciente }\end{array}$} & $\begin{array}{l}\text { Implementação de } \\
\text { protocolos }\end{array}$ & $\begin{array}{l}\text { - Segurança cirúrgica } \\
\text { - Prescrição e administração de medicamentos } \\
\text { - Prevenção de quedas } \\
\text { - Prevenção de ulceras de pressão } \\
\text { - Prescrição, uso e administração de } \\
\text { medicamentos } \\
\text { - Uso de equipamentos e materiais } \\
\text { - Prevenção e controle de eventos adversos } \\
\text { - Terapias enteral e parenteral } \\
\text { - Comunicação efetiva } \\
\text { - Participação dos pacientes e familiares na } \\
\text { assistência prestada } \\
\text { - Estratégias para a promoção do ambiente } \\
\text { seguro }\end{array}$ \\
\hline & $\begin{array}{l}\text { Ações relacionadas } \\
\text { aos riscos e } \\
\text { eventos adversos }\end{array}$ & $\begin{array}{l}\text { - Monitoramento } \\
\text { - Avaliação } \\
\text { - Tratamento } \\
\text { - Comunicação }\end{array}$ \\
\hline
\end{tabular}

Tabela 1: Dimensões mensuráveis quanto à Segurança do Paciente. Elaborado pelos autores com base no Instrumento de Avaliação da Qualidade da EBSERH. 
A coleta de dados ocorreu durante o mês de setembro de 2016 e foi conduzida por meio de visualização direta das condições apresentadas tanto na estrutura quanto nos processos de trabalho das equipes da Unidade de Clínica Médica do Hospital de Ensino. Cada variável do formulário foi minuciosamente observada e detalhada de forma descritiva, para que não houvesse precipitação na análise, tendo em vista a segurança e efetividade da pesquisa.

\section{Análise dos Dados}

Os dados foram registrados em planilhas eletrônicas no programa Microsoft Excel®, versão 2013, para formação de um banco de dados e tabulação.

Para a identificação do risco foi realizado um levantamento dos fatores contribuintes para a ocorrência de incidentes e/ou eventos adversos, através da observação direta e as informações resultantes da análise de dados realizados no diagnóstico situacional.

A análise de risco e definição das possíveis causas associadas aos incidentes/eventos encontrados foram avaliadas através do método de "brainstorming" e o diagrama de Ishikawa. Buscando assim, a identificação da causa fundamental de um processo durante a coleta de dados 6 .

Com base nos padrões estabelecidos pela JCI foi estabelecido o Índice de Conformidade (IC) ideal $\geq 80 \%$ de conformidade para todos os indicadores descritos, ou seja, o percentual de adequação que reflete a segurança em relação à assistência prestada ${ }^{5}$. O IC ideal $(\geq 80 \%)$ foi balizado no Índice de Positividade (IP), que estabelece os seguintes parâmetros: $100 \%$ de positividade significam que para o quesito analisado houve $100 \%$ de acerto ou conformidade, o que corresponde a uma assistência desejável; de 99 a 90\% assistência adequada; de 89 a 80\% assistência segura; de 79 a 70\% assistência limítrofe e menor que 70\% assistência indesejada ou sofrível ${ }^{7}$.

\section{Aspectos Éticos}

Esta pesquisa está ancorada ao "Projeto Qualis: Análise de Indicadores e Processos para Gestão de Qualidade" e foi desenvolvida conforme as normas preconizadas na Resolução do Conselho Nacional de Saúde/CNS número 466/12, que disciplina a pesquisa com seres humanos. O projeto de pesquisa foi submetido ao Comitê de Ética de Pernambuco (CEP/CCS/UFPE) e aprovado conforme Parecer de № 1.763.733 de 06 de Outubro de 2016.

\section{RESULTADOS E DISCUSSÕES}

O Hospital onde o estudo foi realizado possui capacidade instalada de 407 leitos, numa área construída de $62.000 \mathrm{~m}^{2}$. A estrutura predial do Hospital é em formato de $\mathrm{H}$, está divido em 5 blocos, onde estão os blocos B (ao sul) e C (ao norte) constituído de onze andares. O serviço de internação funciona do $4^{\circ}$ ao $11^{\circ}$ andar nos dois blocos, B e C, incluindo as Unidades de Terapia Intensiva (UTI).

O Hospital conta com um Núcleo de Segurança do Paciente (NSP), constituído formalmente em Julho de 2014, seguindo as normatizações da Portaria/MS N529, de $1^{\circ}$ de Abril de 2013 que 
institui o Programa Nacional de Segurança do Paciente (PNSP) e de Resolução da Diretoria Colegiada, RDC N³6, de 25 de Julho de 2013, que institui ações para segurança do paciente em serviços de saúde, com o intuito de promover e apoiar a implementação das metas de segurança, a fim de reduzir, a um mínimo aceitável, o risco de dano desnecessário associado à atenção à saúde ${ }^{8}$.

A Unidade de Clínica Médica (UCM) deste Hospital foi escolhida para sediar o projeto piloto elaborado pelo NSP estando localizada na parte frontal do Hospital, no $11^{\circ}$ andar, possui 13 enfermarias e 26 leitos.

Há uma série de razões para uma organização hospitalar utilizar questionários para avaliar questões relacionadas à cultura de segurança: questionários podem ser utilizados como uma ferramenta diagnóstica para auxiliar na identificação de áreas as quais necessitam melhorias; são muito adequados para avaliar iniciativas de segurança do paciente, avaliam mudanças ao longo do tempo e fazem comparações com referências de dados externos ${ }^{1}$.

A avaliação da cultura é essencial, porque são os hábitos (dos profissionais) que determinam as ações que vão ocorrer em todos os processos, possibilitando identificar e gerir questões relevantes de segurança nas rotinas e condições de trabalho. Esta abordagem permite acessar informações dos funcionários a respeito de suas percepções e comportamentos relacionados à segurança, identificando pontos fracos e fortes de sua cultura de segurança e as áreas mais problemáticas para que se possa planejar e implementar intervenções ${ }^{1}$.

Neste sentido, o formulário traz um capítulo exclusivo sobre a cultura organizacional, com o intuito de conhecer as fragilidades e potencialidades assistenciais que a unidade oferece.

\section{Análise dos Indicadores do Plano de Segurança do Paciente}

A análise dos indicadores do PSP da instituição se deu a partir da leitura do Plano de Segurança do Paciente da Instituição, tendo uma ampla percepção sobre as estratégias utilizadas para definição dos mesmos. As estratégias são estabelecidas pela RDC № 36/2013 da Anvisa ${ }^{8}$.

A UCM ainda não implantou nenhum indicador numérico. Assim, os resultados descritos nesta pesquisa estão baseados na observação direta dos processos de trabalho, das instalações físicas, de informações obtidas diretamente dos profissionais da UCM e da equipe do NSP, que teve como base os registros de Eventos Adversos (EA) do VIGIHOSP (Sistema Eletrônico de Vigilância).

Em 2014, com a formação do NSP, composto por 4 (quatro) enfermeiros exclusivos e destas 2 (dois) podem participar nas instâncias deliberativas do serviço de sáude, iniciou-se o processo de implantação das metas de segurança, formando subgrupos multiprofissionais para trabalhar com cada uma das metas estabelecida na UCM. A equipe tem autonomia para planejar e executar as ações, mas entraves impediram que as metas fossem efetivadas de forma mais rápida, dentre eles estão: os recursos humanos e financeiros; sendo justificado pela transição de gestão que ocorreu neste mesmo período de tempo.

Apesar das barreiras enfrentadas o NSP deu continuidade às atividades referentes à implantação das metas, porém só em 2016 é que pode iniciar com a gerência de risco, a partir da instituição da Unidade de Gerenciamento de Riscos Assistenciais (UGRA), com uma equipe composta por 3 (três) enfermeiros. A UGRA, em conjunto com outras instâncias do hospital, definiu os indicadores para cada meta internacional de segurança do paciente. 
Os Indicadores relacionados à meta 1 "Identificação correta do paciente" foram: Número de eventos adversos devido a falhas na identificação do paciente e proporção de pacientes com pulseiras padronizadas entre os pacientes internados. Este processo assegura ao paciente que a ele é destinado determinado tipo de procedimento ou tratamento, prevenindo a ocorrência de erros e enganos que o possam lesar ${ }^{9,10}$.

De acordo com o Protocolo de Identificação Correta do Paciente do Ministério da Saúde ${ }^{11}$, erros de identificação do paciente podem ocorrer, desde a admissão até a alta do serviço, em qualquer fase do tratamento. Alguns fatores podem potencializar os riscos na identificação do paciente como: estado de consciência do paciente, mudanças de leito, setor ou profissional dentro da instituição e outras circunstâncias no ambiente.

Cerca de 850 pacientes nos Estados Unidos são transfundidos com sangue destinados a outros pacientes e aproximadamente 3\% desses pacientes evoluem para óbito. Em cada 1.000 pacientes que recebem transfusões de sangue ou de hemocomponentes, um indivíduo recebe o que seria destinado à outra pessoa ${ }^{12}$. Neste estudo, os autores ressaltam os altos níveis de consciência profissional da equipe, assim como a importância da participação do paciente para minimizar o risco de dados errôneos e a preocupação com o uso do dispositivo em algumas circunstâncias clínicas especiais, como transfusão de sangue e administração de medicamentos ${ }^{12}$.

Esta é uma meta que já esta implantada na UCM e, durante o período da pesquisa, já havia adotado o uso das pulseiras e a placa de identificação no leito. A responsabilidade de fazer a identificação do paciente, através da pulseira, é do Serviço de Admissão e Alta (SAA). Porém, alguns pacientes tem chegado à UCM sem a identificação e/ou feito um fluxo contrário ao protocolo de internação, sendo admitido diretamente na UCM sem antes passar pelo SAA.

Algumas campanhas educativas intituladas de "quem identifica corretamente o paciente levanta a mão; pulseira de identificação mais segurança para todos" realizadas na instituição como um todo, tem levado a uma adesão dos profissionais de forma contínua, sendo possível observar a mudança de cultura em um pequeno espaço de tempo, de forma que os pacientes/clientes internados na UCM, estavam orientados quanto ao processo identificação.

Os indicadores relacionados à meta 2 (dois) "Melhorar a comunicação entre profissionais de saúde". A comunicação permeia todas as atividades que integram a assistência ao paciente. Quanto mais especializado for o serviço, maior a necessidade de informações técnicas, especializadas e precisas. Por isso foram avaliados: número de eventos adversos devido a falhas na comunicação verbal; taxa de ordens verbais registradas entre o total das informações verbais; e, assegurar comunicação inequívoca em $100 \%$ das prescrições e resultados de exames verbais.

Um requisito essencial para a continuidade do cuidado e a segurança do paciente é a comunicação consistente entre os profissionais, de uma equipe para a próxima e entre profissionais, paciente e familiar. Enquanto os profissionais se alternam, o paciente e a família são os mesmos e, nessa perspectiva, estão em posição-chave para, em parceria com a equipe, assegurar a continuidade do cuidado ${ }^{13}$.

A comunicação efetiva é bidirecional. Para que ela ocorra com segurança, é necessário que haja resposta e validação das informações emitidas. A técnica "leia de volta" (read-back), ou repita o que foi dito, pode ser utilizada, por exemplo, para validar as informações transmitidas na passagem de plantão entre os turnos de trabalho: o profissional anota a informação recebida e repete para a pessoa que a transmitiu, de modo a confirmar que a compreendeu corretamente ${ }^{14}$.

Os resultaram mostraram que esta meta ainda não estava implantada, contudo, foram observadas que ações desenvolvidas para sua efetiva implantação. O protocolo referente à Comunicação Efetiva entre os profissionais ainda estava em fase de avaliação pela gerência e alguns cuidados especiais podem ser destacados: resultados de exames e prescrição médica recebidos 
verbalmente, por telefone; notificar no sistema eletrônico de vigilância (VIGIHOSP) qualquer incidente relacionado ao fornecimento equivocado de informação verbal, quer seja relativo à resultados errados de exames laboratoriais, quer seja erros na administração de medicação; notificar no VIGIHOSP a ausência de registro escrito da informação fornecida verbalmente. Sendo os responsáveis por estas ações os enfermeiros e médicos.

A UCM não tem registros de eventos adversos relacionados à comunicação verbal, contudo foram observadas fragilidades na passagem de plantão.

Os indicadores relacionados à meta 3 (três) "Melhorar a segurança na prescrição, no uso e na administração de medicamentos". Os erros de medicação podem ocorrer em qualquer uma das três etapas do processo de uso de medicamentos: prescrição, dispensação e administração. Assim sendo, os parâmetros avaliados foram: Taxa de erros na prescrição de medicamentos; Taxa de erros na dispensação de medicamentos; Taxa de inadequação dos carros de medicações de urgência.

A UCM não possui protocolo referente ao uso seguro de medicamentos, ficando assim a unidade em estado de alerta, exigindo os profissionais um nível de atenção ainda maior quanto ao uso, guarda e administração dos medicamentos. Porém, ações vêm sendo implementadas no sentido de garantir a segurança e a qualidade na assistência prestada, como por exemplo, a equipe de farmacovigilância que atua com ações de educações referentes à prescrição correta. As principais ações são referentes ao carimbo, pois muitas prescrições chegam sem o carimbo do solicitante; assinatura, muitos esquecem de assinar a prescrição; e a dosagem elevada de alguns fármacos.

Os indicadores da meta 4 (quatro) "assegurar a cirurgia em local de intervenção, procedimento e paciente corretos" são: Percentual de pacientes que recebeu antibiótico profilaxia no momento adequado; Número de cirurgias em local errado; e, Número de cirurgias em paciente errado.

Em outubro do ano de 2004 a Organização Mundial de Saúde (OMS) lançou a "Aliança Mundial para Segurança do Paciente", que aponta à conscientização para melhorar a segurança dos cuidados em saúde, além do desenvolvimento de políticas e estratégias na atenção à saúde. Um dos 'Desafios Mundiais para a Segurança do Paciente', que pretende identificar os itens mais significativos do risco à segurança do paciente, é 'Cirurgia Segura Salva Vidas'. Este desafio foi implementado em 2007 e 2008 para reduzir a ocorrência de danos ao paciente cirúrgico e definir padrões de segurança que podem ser aplicados a todos os países membros da OMS ${ }^{15}$.

Esta é uma meta já implantada no hospital, o que reflete positivamente na UCM. Observou-se a existência de um protocolo eficiente com relação à cirurgia segura e também o uso do cheklist para cirurgia segura. A adoção desta prática reflete o desejo de mudança da instituição e o compromisso com a segurança do paciente. Ressalta-se que mesmo tendo protocolo de cirurgia segura e os usos de checklist, erros podem ocorrer devido a falhas na comunicação, já que esta ainda é uma fragilidade da instituição.

Os indicadores da meta 5 (cinco) "Higienização das mãos para prevenção de infecções relacionadas a assistência a saúde" são: Assegurar 100\% higiene das mãos em todas as oportunidades. Percentual de infraestrutura adequada: número de pontos para higiene.

As mãos compõem a fundamental via de transmissão de microrganismos durante os cuidados de saúde, pois a pele é um possível reservatório de diversos microrganismos, que podem se transferir de uma superfície para outra. A higienização das mãos é a medida individual mais simples e menos dispendiosa para prevenir a propagação das infecções relacionadas à assistência à saúde ${ }^{16}$.

A UCM apresenta 31 pontos de higienização distribuídas nas 13 enfermarias, dos quais 18 são dispensadores de álcool gel e 13 pias. No período da coleta de dados para este estudo, 13 pontos 
dos dispensadores estavam sem álcool gel e 12 pias estavam com água. Apenas uma enfermaria não tinha torneira na pia.

Neste sentido, não tem como prever uma meta de $100 \%$ de higienização das mãos, uma vez que a UCM apresenta problemas estruturais, o que dificulta a adesão de tais práticas, possibilitando o surgimento de infecções cruzadas, podendo assim aumentar o tempo de permanência na unidade de saúde, gerando mais desconfortos aos pacientes/clientes, aumentando os custos assistenciais e diminuindo a rotatividade de leitos.

Os indicadores da meta 6 (seis) "Reduzir o risco de quedas e úlceras por pressão" são: Identificar $100 \%$ dos pacientes com risco para queda e úlcera cutânea e aplicar medidas de prevenção; número de quedas com dano; número de quedas sem dano.

As úlceras por pressão (UPP) são consideradas evitáveis e, em muitos casos, graças à adoção de medidas de prevenção, mas representam um grande desafio para a assistência em saúde, representando alto custo financeiro e emocional para os pacientes/clientes, familiares e para as organizações de saúde ${ }^{17}$. Ainda que seja considerado um problema de alta incidência em pacientes hospitalizados e classificado como um EA, a sua ocorrência nem sempre está condicionada a falhas no cuidado, mas, por vezes, a questões intrínsecas dos pacientes atendidos ${ }^{18}$.

Esta meta ainda não está implementada. Algumas ações já estão sendo desenvolvidas e com resultados positivos, como a escala de Braden, que tem sido usada na UCM e o relógio que permite a interação do acompanhante na mudança de decúbito.

A adesão à escala de Morse para identificar pacientes com risco de quedas, devido a algumas barreiras estruturais ainda não está implantada.

Vale ressaltar que a UCM não apresentou nenhuma incidência de UPP, durante todo período de coleta, bem como não houve registro de quedas com danos ao paciente. Alguns registros de quedas sem danos foram encontrados no VIGIHOSP, todos associados a idosos que ficaram sem acompanhantes em algum momento durante o período de internação em outras unidades.

Os indicadores da meta 7 (sete) "Melhorar a segurança na prescrição das solicitações de transfusões de sangue (STS), no uso e na administração de hemocomponentes" são: Identificar corretamente $100 \%$ das amostras; e, Preencher corretamente $100 \%$ das Solicitações de Transfusões de Sangue (STS).

A transfusão de sangue e hemocomponentes são indicadores para pacientes que sofreram perda sanguínea significante ou alterações hematológicas decorrentes de doenças ou procedimentos. Algumas ações são sugeridas para evitar erros na administração, pois podem comprometer a segurança do paciente ${ }^{19}$.

No Brasil, os órgãos e os serviços responsáveis por transfusões de sangue, pelo controle e prevenção da infecção associada ao cuidado em saúde e pelos serviços de anestesia podem ser considerados pioneiros no que tangem as medidas que promovem a segurança do paciente.

Estes, há anos, adotam medidas para garantir a segurança dos processos de cuidado, com bons resultados ${ }^{20}$.

Esta é uma meta que ainda não foi implementada, contudo já existe protocolo de excelência dentro da instituição, o que não mitiga os erros nas solicitações de transfusões de sangue (STS), mas ao chegar ao serviço de hemoterapia as fichas voltam à unidade solicitante para as devidas correções, impossibilitando assim que erros aconteçam. 
Considerando todos os parâmetros avaliados quanto a estruturação do Núcleo de Segurança do Paciente na UCM e a implantação dos indicadores relacionados as metas Internacionais de Segurança do Paciente, observou-se um ìndice de Conformidade (IC) de 56,25\%, ou seja, inferior ao recomendado pela $\mathrm{JCl}(\geq 80 \%)$.

\section{Avaliação dos riscos inerentes aos processos de trabalho no setor de clínica médica}

O mapeamento dos riscos inerentes aos processos de trabalhos na UCM ainda está em processo de implantação. A Avaliação Preliminar dos Riscos (APR) assistenciais na unidade já foi construída e encaminhada para as chefias.

Em 1944, foi criada a primeira legislação estabelecendo a obrigatoriedade de formação das Comissões Internas de Prevenção de Acidentes (CIPA). Contudo, os acidentes de trabalho continuaram a crescer, com grandes perdas humanas e econômicas.

O mapa de riscos é a representação gráfica dos riscos de acidentes nos diversos locais de trabalho. Risco é definido por toda e qualquer possibilidade de que algum elemento ou circunstância existente num dado processo ou ambiente de trabalho possa causar dano à saúde, seja por meio de acidentes, doenças ou do sofrimento dos trabalhadores, ou ainda por poluição ambiental $^{21}$. Os riscos podem ser classificados em: físicos; químicos; biológicos; ergonômicos.

Desta forma, na UCM acidentes podem ocorrer em função da não existência do mapa de risco, tornando assim o ambiente susceptível a falhas. Contudo, ressalta-se que erros são esperados mesmo nas organizações de excelência, pois errar é inerente ao ser humano e não é possível muda-lo, mas é possível mudar as condições em que os indivíduos trabalham, podendo assim mitigar os erros e até preveni-los.

A implementação de estratégias para promoção do ambiente seguro, através do SOST (Serviço de Saúde Ocupacional e Segurança do Trabalho) e da Comissão Interna de Prevenção de Acidentes (CIPA) foram observadas.

\section{Análise das estratégias de segurança do paciente implantadas no setor, sob a perspectiva da dimensão do cuidado de saúde.}

No contexto organizacional, as estratégias são de importância para qualquer organização seja empresarial visando o lucro e enfrentar de forma positiva as concorrentes, quanto hospitalar na busca de uma qualidade assistencial.

Desta forma, pode-se afirmar que as estratégias podem ser consideradas como uma mola impulsora para uma gestão de qualidade; no âmbito hospitalar, elas tomam uma ênfase ainda mais complexa, pois além de buscar garantir o sucesso dos planos e métodos assistenciais, elas visam uma integração de toda rede de saúde com o objetivo de garantir a SP.

Até os anos 1990, o foco da saúde estava no aspecto curativo. Hoje, os sistemas de saúde estão voltados para a prevenção. Essa mudança de enfoque, associado às novas tecnologias, à conscientização do paciente, à crise de financiamento e à falta de profissionalização da gestão, são situações que têm feito com que os hospitais tentem alterar suas estratégias de gestão para poderem sobreviver em cenário tão adverso ${ }^{3}$.

Para uma assistência segura faz-se necessário um planejamento no qual sejam definidas as estratégias que impliquem diretamente na qualidade da assistência. Devem ser levados em 
consideração a implementação de protocolos, o mapeamento dos riscos assistenciais, as barreiras que impeçam a comunicação efetiva, a mitigação de EA e a mudança da cultura organizacional, que acompanhe os preceitos da segurança do paciente.

A segurança do paciente resulta do esforço e comprometimento diário de equipes multiprofissionais, instituições e serviços de atenção à saúde, públicos e privados, de processos e sistemas organizados, avaliados e aprimorados continuamente quanto à prevenção e redução de danos, do reforço contínuo para as boas práticas assistenciais recomendadas por agências nacionais e internacionais, da formação de profissionais da saúde e de uma política nacional de segurança no cuidado à saúde ${ }^{22}$.

Sobre os processos de Gestão de Risco relacionados às Tecnologias em Saúde, considerando as ações e serviços desenvolvidos pelo NSP dentro da unidade hospitalar, observou-se um percentual de $60,0 \%$ de conformidade. O padrão de conformidade instituido pela $\mathrm{JCl}$ para se ter uma assistência segura é de $\geq 80 \%$.

Os processos de gestão de riscos relacionados às tecnologias estão alocados no SCIH (Serviço de Controle da Infecção Hospitalar) e vinculados a Gerência de Atenção a Saúde, da Instituição Hospitalar, executando as açoes de tecnovigilância. A engenhraria clínica realiza as ações de manutenção preventiva e corretiva dos equipamentos e a equipe de enfermagem faz a checagem dos equipamentos, mas não foram observados documentos que formalizem estas atividades.

O SCIH também executa ações de farmacovigilância que é definida como conjunto de procedimentos para o monitoramento das reações transfusionais resultantes do uso terapêutico de sangue e seus componentes, visando a melhoria da qualidade dos produtos e processos em hemoterapia e o aumento da segurança do paciente.

Sobre o serviço de hemovigilância e reações transfusionais e demais incidentes, foi observado um padrão de qualidade adequado, inclusive com indicadores de acompanhamento destas ações já implantados.

\section{Avaliação da Cultura Organizacional}

A cultura é um processo contínuo de desenvolvimento social de uma coletividade adiquirido através da aprendizagem, pela qual as competências, habilidades, conhecimentos, comportamentos e valores são adiquiridos ou modificados, como resultado do estudo, experiência, formação, raciocínio e observação. Desta forma ela é dinâmica e torna-se um elemento adaptativo, podendo sofrer mudanças, ganhando novos traços e perdendo alguns ${ }^{23}$.

A cultura organizacional é positivamente correlacionada com o comportamento do líder. O líder influencia no desenvolvimento de comportamentos, valores e crenças de seus subordinados, possibilitando o fortalecimento da cultura organizacional ${ }^{1}$.

Melhorias na segurança do paciente são conseguidas com sucesso quando as instituições de saúde constituem e incorporam uma cultura de segurança.

Uma cultura de segurança promove uma aprendizagem na organização, onde os membros da equipe repartem informações sobre os erros a fim de precaver a sua recorrência. A instituição deve ressaltar que a responsabilidade é partilhada, o que significa que todos têm responsabilidade pela segurança do paciente, independente do cargo. Pacientes e familiares devem ser incluídos como parceiros respeitados e devem entender a sua própria responsabilidade, para manterem-se seguros $^{24}$. 
Com base na observação direta da equipe do NSP na UCM, observou-se $60,97 \%$ de conformidade sobre os parâmetros relacionados à cultura de segurança do paciente na organização, quando o ideal deve ser $\geq 80 \%$.

Observa-se a necessidade da implementação de medidas que assegurem a instituição de uma cultura voltada para a segurança do paciente, identificando erros, adequando processos e promovendo melhorias e incentivos, na busca pela qualidade da assistência à saúde.

Diversos autores expressam a qualidade da atenção hospitalar afirmando que muitos pacientes dentro dos hospitais sofrem danos evitáveis e eventos adversos ocasionados durante o processo assistencial e que os mesmos se constituem um problema de saúde pública, necessitando de respostas urgentes e efetivas para sua redução em serviços de saúde ${ }^{13,25-27}$.

Apesar de ter sido instituído em 2014, o NSP só veio a ter maior ênfase e resultados nas ações em 2016, desta forma acredita-se que as fragilidades encontradas sejam mitigadas de forma mais rápida e efetiva, passando assim a utilizar os indicadores como forma de contribuir para melhoria continua da organização.

Iniciativas exitosas foram observadas na instituição como, por exemplo, o programa de capacitação em segurança do paciente dos funcionários do hospital; a campanha "adorno zero"; o treinamento para uso do VISHOSP; treinamento de biosegurança; e, difusão da campanha de identificação do paciente.

Grande parte destas ações são desenvolvidas entre o NSP, a UGRA, a SCIH e o NEPE (Núcleo de Educação Permanente).

Compreende-se que a mudança organizacional do processo de cuidado assistencial, permitirá o rompimento com as práticas tradicionais e possibilitará a permuta das condutas individuais para as coletivas.

\section{CONSIDERAÇÕES FINAIS}

O presente estudo avaliou o padrão de cumprimento das normas de segurança do paciente na clínica médica de um hospital de ensino em Pernambuco. Por meio da observação dos processos de trabalhos e a utilização do formulário da EBSERH, constatou-se alguns entraves no processo de efetivação da implantação do NSP. Contudo, tais entraves não impediram a continuidade das ações programadas para a UCM da instituição em foco, compreendendo que é um processo novo, não habitual aos serviços públicos de saúde e com resultados de longo prazo.

Foi possível mensurar os riscos inerentes aos processos de trabalho, tendo como base as metas internacionais definidas pela Joint Commission International. Apesar da UCM já está com a UGRA instalada, o mapa de risco ainda não foi aprovado pela gerência geral, ele serve para mostrar os riscos presentes no ambiente de trabalho, fazendo um diagnóstico da situação do setor, como também, para determinar medidas de prevenção ou anulação dos referidos riscos.

A ausência de protocolos essenciais como o de higienização das mãos, de administração e guarda de medicamentos, de prevenção de quedas e de UPP (em apreciação), são alguns protocolos relacionados às metas de segurança da $\mathrm{JCl}$.

Outra crítica importante feita à análise dos riscos é a estrutura física que necessita de algumas reformas com urgências, de forma que as possibilidades de riscos provenientes da mesma possam não existir, como: melhorias nos pisos, evitando quedas; pontos de higienização evitando 
as IRAS, pois muitos profissionais saem da anamnese e não realizam assepsia das mãos e nem dos equipamentos utilizados.

Através dos riscos mensurados e as estratégias de segurança do paciente analisadas, será possível elaborar um plano de intervenção, utilizando para isso as ferramentas de gestão apropriadas.

\section{REFERÊNCIAS BIBLIOGRÁFICAS}

1. Reis, C. T.; Martins, M.; Laguardia, J. A segurança do paciente como dimensão da qualidade do cuidado de saúde: um olhar sobre a literatura. Ciência \& Saúde Coletiva, v. 18, n. 7, p. 20292036, 2013. Disponivel em: http://www.scielo.br/pdf/csc/v18n7/18.pdf acesso em 25 de maio de 2016.

2. Brasil. Agência Nacional de Vigilância Sanitária (ANVISA). Cirurgias Seguras Salvam Vidas. Fôlder, Brasília, 2008. Disponível em:

http://www20.anvisa.gov.br/segurancadopaciente/index.php/noticias/60-cirurgiasseguras-salvamvidas acesso em 25 de outubro de 2016.

3. Kohn, Linda T.; Corrigan, Janet M.; Donaldson, Molla S. To err is human: Building a Safer Health System. Editors Committee on Quality of Health Care in America, Institute of Medicine, National Academy Press, Washington, D.C. 1999. http://www.csen.com/err.pdf Acesso em 25 de abril de 2016.

4. Aline, A.; Bueno, B.; Fassarella, C. S. Segurança do Paciente: uma reflexão sobre sua trajetória histórica Patient Safety: a reflection on its historical trajectory. Revista Rede De Cuidados De Saúde, p. 2-10, 2011. Disponível em:

http://publicacoes.unigranrio.edu.br/index.php/rcs/article/view/1573 acesso em 28 out de 2016.

5. JCl. Joint Commission International Accreditation Standards for Hospitals: Standards Lists Version, Region Hovedstaden, 2011. Disponível em:

http://www.jointcommissioninternational.org/common/pdfs/jcia/IAS400 Standards Lis ts Only.pdf

Acesso em 11 novembro 2011.

6. Feltraco, Elcídio Jose; Soares, Luciano Elias; Gomes, Willian Bianchi; Lizote, Suzete Antonieta; Verdinelli, Miguel Angel; Lana, Jeferson. Análise da adoção de normas para a qualidade ISO 9001: um estudo de caso com base no ciclo PDCA na visão dos envolvidos no processo. NAVUS, Revista de Gestão e Tecnologia, p. 43-56, 2012.

7. Saupe, R. Horr, L. Auditoria em enfermagem. Revista de Ciências da Saúde. Florianópolis, v.1, n.1, p.23, 1982.

8. Brasil. Ministério da Saúde. RDC No 36 , de 25 de julho de 2013. Brasília, 2013. Disponivel em: http://www20.anvisa.gov.br/segurancadopaciente/index.php/legislacao/item/rdc-36de-25-de-julhode-2014 acesso em 25 de out de 2016.

9. Gurgel, L. et al. Artigo Análise Reflexiva Contribuição De James Reason Para a Segurança Do Paciente : Reflexão Para a Prática De Enfermagem Contribution of James Reason To Patient Safety: Reflection for Nursing. v. 8, p. 2507-2512, 2014.

10 .Chang, P. et al. O processo de acreditação JCl : diálogo com a gestão de Acreditação , Padrões e Mensuração The $\mathrm{JCl}$ accreditation process : dialogue with the Accreditation management, Standards and Measurement Entrevista com Paul Chang. v. 9, p. 133-140, 
2015.Disponivel em: file://C:/Users/josisilva/Downloads/215-1263-1-SM.pdf acesso em 21 de outubro de 2016.

11. Brasil. Ministério da Saúde. Portaria No 529, de 10 de abril de 2013. Diário Oficial da União, v. 2013, p. 43-44. Brasília, 2013. Disponível em:

file:///C:/Users/josisilva/Downloads/portaria\%20ms\%20n\%20529\%202013\%20\%20segurana\%20d o\%20paciente.pdf acesso em 28 de out de 2016.

12. Smith, A.F., Casey K, Wilson J; Fischbacher-Smith D. Wristbands as aids to reduce misidentification: an ethnographically guided task analysis. Int J Qual Health Care. 2011 Oct;23(5):590-9.

13. Gonçalves, M. I. et al. Comunicação e segurança do paciente na passagem de plantão em unidades de cuidados intensivos neonatais. Texto \& Contexto Enfermagem, Florianópolis, SC, v.25, n.1, p. e2310014. 2016.

14. REBRAENSP. Estratégias para a segurança do paciente : manual para profissionais da saúde. Disponivel em:< Disponível em: http://www.rebraensp.com.br/pdf/manual seguranca paciente.pdf acesso em 11 de novembro de 2016.

15. Pancieri, A. P. et al. Checklist de cirurgia segura: análise da segurança e comunicação das equipes de um hospital escola. Rev. Gaúcha de Enfermagem, v. 34, n. 1, p. 71-78, 2013. disponível em : http://www.scielo.br/pdf/rgenf/v34n1/09.pdf acesso em 26 de out de 2016.

16. Lima, F. D. M. a Segurança Do Paciente E Intervenções Para a Qualidade Dos Cuidados De Saúde. p. 22-29, 2011. Disponível em:

http://www.uel.br/revistas/uel/index.php/espacoparasaude/article/view/18190 acesso em 24 de outubro de 2016

17. Lucena, A. D. F. et al. Perfil clínico e diagnósticos de enfermagem de pacientes em risco para úlcera por pressão. Rev. Latino-Am. Enfermagem, v. 19, n. 3, p. 1-8, 2011. Disponível em:

http://hdl.handle.net/10400.26/13214 Acesso 15 de maio de 2016.

18. Medeiros, A. B. F.; Lopes, C. H. A. D. F.; Jorge, M. S. B. Análise da prevenção e tratamento das úlceras por pressão propostos por enfermeiros. Revista da Escola de Enfermagem da USP, v. 43, n. 1, p. 223-228, 2009.

19. COREN/SP; Conselho Regional de Enfermagem do Estado de São Paulo. 10 Passos para a Segurança do Paciente. Conselho Regional de Enfermagem Sao Paulo, p. 32, 2010. Disponível em: http://www.corensp.gov.br/sites/default/files/10 passos seguranca paciente 0.pdf. Acesso em 26 de outubro de 2016

20. Brasil. Ministério da Saúde. RDC N³4, DE 11 DE JUNHO DE 2014. Dispõe sobre as Boas Práticas no Ciclo do Sangue. Brasília, 2014. Disponível em: http://www.hemope.pe.gov.br/pdf/3006-2016/06-rdc-34-de-11-06-2014.pdf Acesso 15 de maio de 2016.

21. Porto, M. F. de S. Análise de riscos nos locais de trabalho: conhecer para transformar. p. 41, 2000. Disponível em

https://normasregulamentadoras.files.wordpress.com/2008/06/riscos trabalho.pdf

22. Harada, M. D. J. e Pedreira, M. Assistência Segura: Uma Reflexão Teórica Aplicada à Prática. Segurança do Paciente e Qualidade em Serviços de Saúde, p. 41-55, 2013.

23. Perez, F.C.; Cobra, M. Cultura Organizacional e Gestão Estratégica: A cultura como recurso estratégico. São Paulo: Atlas, 2015. 
24. Sousa, C. S. de O.; da Silva, A. L. O cuidado a pessoas com HIV/aids na perspectiva de profissionais de saúde. Revista da Escola de Enfermagem da USP, v. 47, n. 4, p. 907-914, 2013.

25. Fragata, J. Sessão 7: Traduzir a evidência em Cuidados de Saúde mais Seguros. p. 50, 2012.

26. Duarte, S. da C. M. et al. Eventos adversos e segurança na assistência de enfermagem. Revista brasileira de enfermagem, v. 68, n. 1, p. 136-46, 144-54, 2015. Disponível em: http://www.scielo.br/scielo.php?script=sci arttext\&pid=S003471672015000100144\&lng=en\&nrm=i so Acesso em 22 maio de 2016.

27. Reason, J. Human error: models and management. BMJ, v. 320, n. November 2006, p. 768770, 2000.

Recebido: 04/12/2017; Aceito e publicado: 15/12/2017

Correspondência: Flávia C. Morone Pinto. Centro Acadêmico de Vitória (CAV/UFPE). Vitória de Santo Antão - Pernambuco, Brasil. E-mail: fcmorone@gmail.com

Contribuição dos autores: todos os autores participaram da elaboração do conteúdo, da análise e interpretação dos dados, da redação e da revisão final.

Conflito de Interesses: os autores declaram não haver conflito de interesses.

(C) This is an Open Access article distributed under the terms of the Creative Commons Attribution License, which permits unrestricted use, distribution, and reproduction in any medium, provided the original work is properly cited. 